\title{
There and Back Again: An Unfinished Tale - XEDS in the AEM
}

\author{
Nestor J. Zaluzec ${ }^{1}$
}

1. Photon Sciences Division, Argonne National Laboratory, IL, USA.

For the last 40 years the microanalysis community has benefited from technological innovations which facilitate X-ray Energy Dispersive Spectroscopy (XEDS) in electron column instruments [1,2]. My quest to find the proverbial XEDS/AEM Ring to rule them all started in the Summer of 1973 at the University of Illinois with Hamish Fraser and John Woodhouse and continues today. In hindsight, it is not without surprise that even though a fair amount of time has passed we continue to improve, significantly so, upon the original work done by Lorimer et al [3]. Interfacing detectors to Scanning/Transmission Analytical Electron Microscopes in the early 70's was a built it yourself project, fortunately, the $\mathrm{Si}(\mathrm{Li})$ detectors themselves were a commercial product for low energy beam lines (Electron Microprobes and Scanning Electron Microscopes), but they were not quite ready for prime time operations in a $200 \mathrm{kV} \mathrm{S} / \mathrm{TEM}$ where when we started at the UofI. The geometries and specimen stages employed were restricted by the design of the electron optical columns, substantial restrictions on the detector collimation, subtending solid angles, specimen holders, deleterious effects of uncollimated fluorescing radiation as well as contamination needed to be understood and controlled [3-7]. While a number of these issues have been mitigated today, there still are substantial, albeit, new challenges.

Figure 1 illustrates the evolution of detectors currently used in S/TEM instruments from the early $\mathrm{Si}(\mathrm{Li})$ system with their $3 \mathrm{~mm}$ thick crystals in the early-to-mid 70's (Fig. 1a), to the customized small detectors to fit into constrained pole pieces of the TEM common in the late 80-90's (Fig.1b), and finally to modern ultra-thin SDD chips (Fig. 1c). The former allowing a large range of possible geometries (Fig. 2a-e) which are practical in today's AEM. Importantly, the often quoted "solid angle" of SDD detectors should be always questioned owing to the fact that the values advertised rarely take into account the penumbra effects associated with the detector position and specimen holder design. Calculating and measuring the realizable solid angle which directly impact the analytical sensitivity of a measurement is an important part of today's experiments and is best done using a standard test specimen and protocol [8]. An example of a comparison of modeled versus the experimental variation in the solid angle of a XEDS system in a S/TEM as a function of geometry and holder penumbra is shown in Figure 3, and illustrates the solid angle versus holder orientation/tilt. Region \#1 with tilt > 10 degrees represents the regime of full solid angle performance, region \#2 increasing shadowing of the detector, and \#3 the minimum performing orientation. The details of this variation are dependent on system parameters, which include holder dimensions, as well as detector size and elevation angle. Hypothetically an optimized geometry (figure $2 \mathrm{f}$ ) in excess of 口 $\mathrm{sR}$ is achievable [9].

\section{References:}

[1] Fitzgerald R., Keil, K., Heinrich KFJ. Science 159 (1969), p. 528.

[2] Gatti E.; Rehak P., Nuci. Instr. And Meth. A 225 (1984), p. 608.

[3] Lorimer G., Razik N.A., Cliff, G. J. Microscopy 99(2) (1972), p. 153.

[4] Zaluzec, NJ, Fraser HL, J Phys E. Scientific Instruments 9(12) (1976), p. 1051.

[5] Fraser HL, Zaluzec NJ, Woodhouse JB and Sis LB, Proc. of EMSA, SF Press (1975) p. 106.

[6] Zaluzec NJ and Fraser HL, Proc. of the $11^{\text {th }}$ Conference of the Microbeam Analysis Society (1976) p. 14.

[7] Zaluzec NJ, Introduction to Analytical Electron Microscopy (Plenum Press) (1979), p. 121. 
[8] Zaluzec NJ, Microsc. Microanal. 22(S3) (2016), p. 278.

[9] This work was supported by Office of Science, Contract No. DE-AC02-06CH11357 in the Photon Sciences Division at Argonne National Laboratory.
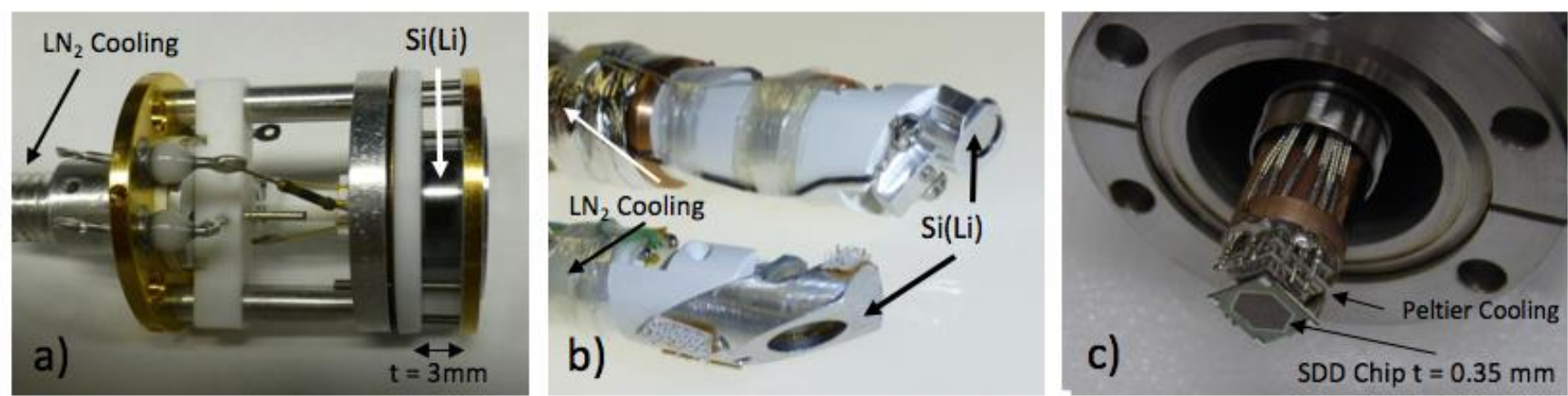

Figure 1. a) Si(Li) circa 1975, b.) Si(Li) 1980-90’s, c.) SDD 2003 used in a modern UHV STEM

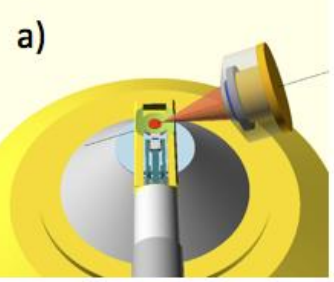

d)

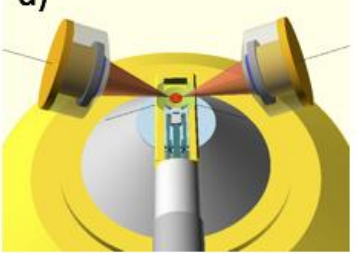

b)

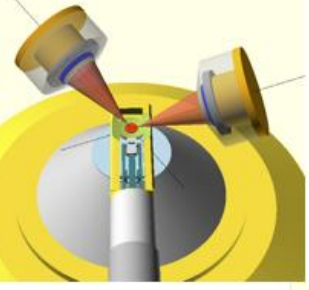

e)

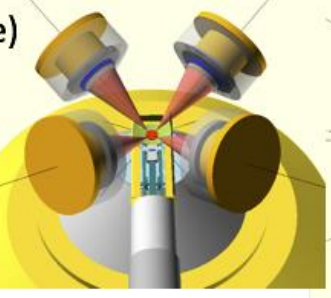

c)

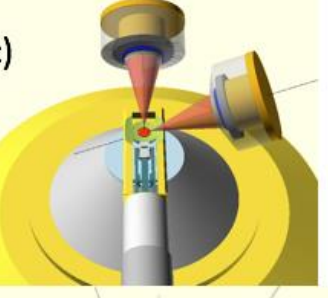

f)

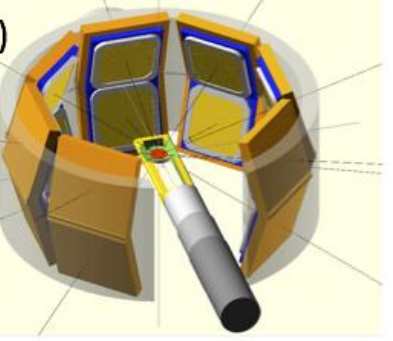

Figure 2. a-e)

Examples of the current XEDS geometries commercially available in today's TEM/STEM instruments

f) Hypothetical space filling geometry to maximize solid angle.
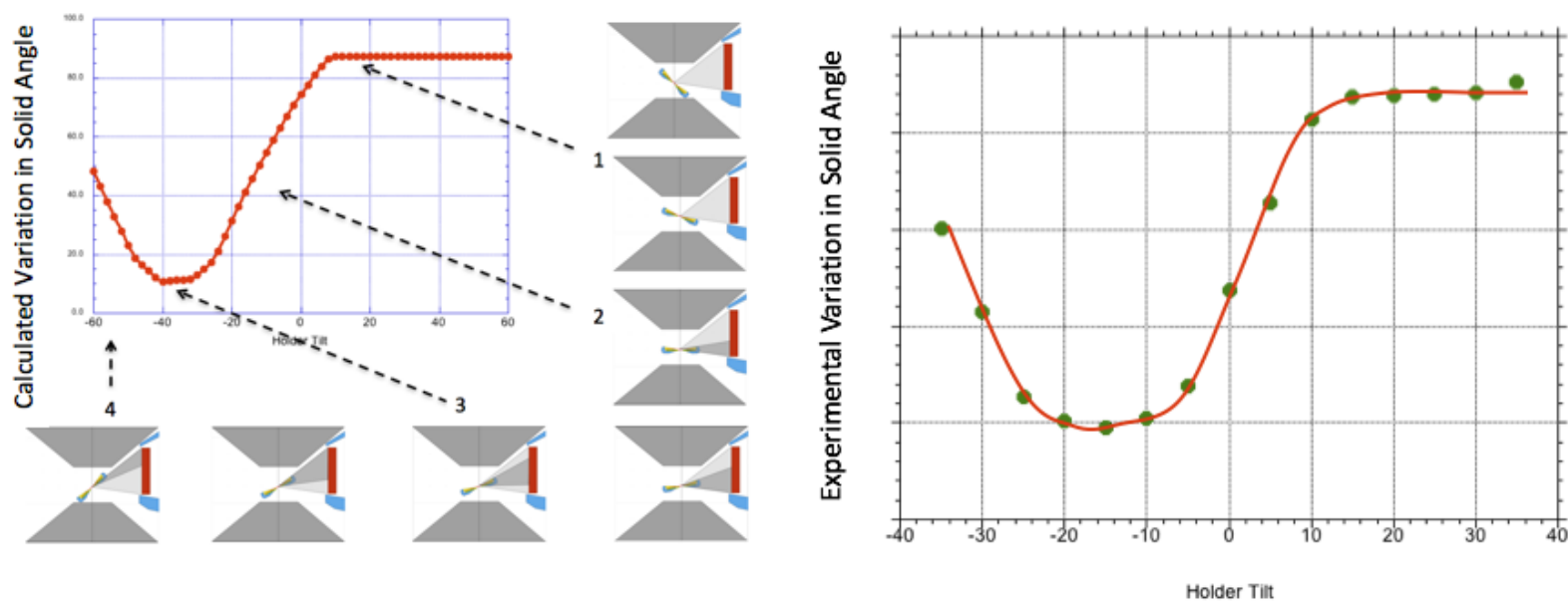

Figure 3. Modeled vs Experimental variation in the realizable solid angle of a XEDS system in a $\mathrm{S} / \mathrm{TEM}$ as a function of geometry and holder penumbra 